\title{
Ocular Weakness in Myasthenia Gravis: Changes in Affected Muscles are a Distinct Clinical Feature
}

\author{
Robert H.P. de Meel ${ }^{\mathrm{a}, *}$, Wouter F. Raadsheer ${ }^{\mathrm{a}}$, Erik W. van Zwet ${ }^{\mathrm{b}, 1}$, Martijn R. Tannemaat ${ }^{\mathrm{a}}$ \\ and Jan J.G.M. Verschuuren ${ }^{\mathrm{a}}$ \\ ${ }^{a}$ Department of Neurology, Leiden University Medical Center, Leiden, The Netherlands \\ ${ }^{\mathrm{b}}$ Department of Biostatistics, Leiden University Medical Center, Leiden, The Netherlands
}

\begin{abstract}
.
Introduction: In this study we quantitatively describe ocular weakness patterns in myasthenia gravis (MG) to help neurologists in making the clinical diagnosis and to investigate how the current outcome measures reflect ocular weakness in MG.

Methods: We investigated ptosis and diplopia patterns in a retro- and prospective cohort of 306 MG patients. Diplopia was systematically examined by testing extra-ocular muscle (EOM) fatigability in two horizontal and four oblique directions for 60 seconds.

Results: Of patients with initial symmetric ptosis, $40 \%$ developed asymmetric ptosis at the second visit. Changes in form of ptosis occurred less often in seronegative MG patients (50\%) than in patients with acetylcholine receptor (AChR) antibodies $(70 \%)$ or muscle-specific kinase (MuSK) antibodies $(69 \%)(p=0.038)$. Of patients with diplopia on the first visit, double vision contained both a vertical and horizontal component in $95 \%$. At the second visit, $83 \%$ manifested diplopia in other gaze directions. The mean time (in seconds) to diplopia was $11.6 \pm 14.0$ and the mean time to ptosis was 27.6 \pm 19.8 . Diplopia or ptosis manifested within 30 seconds in $87 \%$ and 58\%, respectively. Patients who manifested diplopia after 30 seconds, reported no limitations due to diplopia.

Discussion: Changes in the gaze directions in which diplopia occurs or ptosis side occur frequently in MG. In diagnostically challenging cases, we recommend testing ptosis and diplopia in multiple gaze directions for 30-60 seconds during at least two follow-up visits to maximize the chance of observing changes in ocular weakness patterns.
\end{abstract}

Keywords: Myasthenia gravis, ocular weakness, ptosis, diplopia, fluctuations

\section{INTRODUCTION}

Myasthenia gravis (MG) is an autoimmune disease characterized by fatigability and fluctuating muscle weakness that usually begins in ocular muscles [1]. The affected ocular muscles can be subdivided in

\footnotetext{
${ }^{1}$ The corresponding author performed the statistical analysis with the aid of Dr. E.W. van Zwet.

*Correspondence to: Robert H.P. de Meel, Leiden University Medical Center, Department of Neurology, J3-166, P.O. Box 9600, 2300 RC Leiden, The Netherlands. Tel.: +31 71 5262118; E-mail: rhpdemeel@lumc.nl.
}

muscles that move the eyeball (extraocular muscles; EOM), the muscle that elevates the upper eyelid ( $m$. levator palpebrae superioris; LPS) and the muscle involved in closure of the eye (m. orbicularis oculi; OO). Weakness of these muscles results in diplopia, ptosis, and dry eyes, respectively. Weakness of the $\mathrm{OO}$ is less frequent and often occurs later on in the disease course [2]. Although MG is a systemic disease, ptosis is reported to be typically asymmetric. In addition, the (more pronounced) ptosis has been reported to shift from one eye to the other during the disease course, but no quantitative data of 
this phenomenon are reported [3]. Furthermore, different reports describe distinct patterns of diplopia in MG $[4,5]$. Unfortunately, only data from small retrospective cohorts is available and fatigability of EOMs has not been tested systematically. Detailed knowledge of the patterns and fluctuations of ocular weakness in MG is essential for neurologists treating patients with neuromuscular disorders: for diagnosis, to understand its pathophysiology and to establish the relevance of current commonly used outcome measures $[6,7]$. In this study, we therefore aimed to investigate patterns of ptosis, diplopia and eye closure weakness (ECW) in MG patients and the sensitivity of the most frequently used clinical MG outcome measures for EOM weakness in a large prospective cohort of MG patients.

\section{METHODS}

\section{Study design and participants}

We included a prospective cohort of MG patients under treatment at the Leiden University Medical Center between 2016 and 2017 for systematic analysis of ptosis, EOM weakness and ECW. Healthy controls were also included to validate our tests for EOM weakness. In addition, patient records were included of patients under treatment at our hospital retrospectively alongside the prospective cohort to study the occurrence and symmetry of ptosis and ECW over time. As the collection of ptosis and ECW data occurred in the same way for both the prospective and the retrospective cohort (by using the appropriate items from quantitative myasthenia gravis (QMG) scores), the data from both cohorts could be pooled. Retrospective analysis of EOM weakness from patient records was not possible as structured diplopia testing in two horizontal and four oblique directions for 60 seconds was not routinely performed during previous clinical visits. EOM weakness was therefore only tested in a prospective cohort. The diagnosis of MG was based on a combination of clinically confirmed fluctuating muscle weakness and the presence of serum autoantibodies to the acetylcholine receptor (AChR) or muscle-specific kinase (MuSK). Seronegative myasthenia gravis (SNMG) was defined as fatigable muscle weakness in combination with at least one positive rest result: abnormal decrement (at least $10 \%$ ) during low-frequency repetitive nerve stimulation, increased jitter in single-fiber electromyography testing or a positive neostigmine test [1].

\section{Standard protocol approvals, registrations, and patient consents}

The Medical Ethics Boards of the Leiden University Medical Center approved the study and its use of human subjects. All patients provided informed, written consent prior to study participation.

\section{Outcome variables}

In the prospective cohort of MG patients and healthy controls, EOM fatigability was systematically examined by asking patients to avert eye gaze in two horizontal and four oblique directions for 60 seconds. The angles in which eyes were averted were fixed, as the patients were seated in a chair with a fixed position and were instructed to look at dots on a wall. Angles were chosen according to normal ranges of eye movement and with respect to ranges decreasing with age [8-10]. Horizontal angles were 30 degrees and the angles of elevation and depression (for the oblique gaze directions) were 23 and 46 degrees, respectively. These angles were within the normal ranges of elderly persons and were used for all participants. We always ensured dots were seen with both eyes at the start of the test for each particular gaze direction. The provocation time at which diplopia occurred was recorded in seconds. After manifestation of diplopia, patients were asked to cover one eye and asked whether one of the images disappeared in order to rule out monocular diplopia. In addition, ptosis was tested by asking patients to look upwards for 60 seconds. Patients were asked not to use pyridostigmine for at least five hours prior to testing. In the combined proand retrospective cohort, the presence of ptosis and ECW was investigated by collecting data from patient records. Moreover, we analyzed QMG and Myasthenia Gravis Activities of Daily Living (MG-ADL) scores in all MG patients. In addition, the diplopia items of the QMG (first item) and the MG-ADL (seventh item; MG-ADLdip) were independently investigated to assess sensitivity for overall diplopia or the relation between physician-reported EOM weakness and patient-reported limitations of EOM weakness. The relation between physician-reported ptosis (second QMG item) and patient-reported limitation due to ptosis (eighth MG-ADL item; MGADLpto) was also investigated. Additional clinical data collected in this study were sex, age, age at onset of first symptoms and presence of one or more additional autoimmune disease(s) (AID) 
and thymectomy with or without the presence of thymoma.

\section{Statistical analysis}

Kaplan-Meier survival curves were plotted to illustrate the differences in changes of ptosis type over time between MG patients with initial asymmetric or symmetric ptosis. To evaluate the differences between the curves a log-rank test was performed. A chi-squared test was performed to analyse the association of MG-ADLdip with the presence of diplopia and to analyse differences in the occurrence of changes in ptosis between different serological groups (AChR, MuSK or SNMG). Pearson correlation coefficients were used to investigate the correlation between patient-reported burden of diplopia or ptosis (MG-ADLdip or MG-ADLpto) and the shortest time to diplopia (of six gaze directions) or ptosis. $P$-values $<0.05$ were considered significant. Statistical analyses were performed using SPSS version 23 (IBM Corp., Armonk, NY) and GraphPad Prism version 7.00 (GraphPad Software Inc., San Diego, CA).

\section{RESULTS}

\section{Baseline characteristics}

Ptosis and ECW were studied in 306 MG patients, 162 in a retrospective cohort and 144 in a prospective cohort, and 1402 visits were analyzed. Diplopia was investigated in a prospective cohort of 144 MG patients and 20 healthy controls and 210 visits were analyzed. Baseline characteristics of all patients are shown in Table 1.

\section{Ocular weakness patterns}

In our complete cohort ptosis was present on the first visit in $66 \%$ and ECW in $28 \%$. In our prospective cohort, diplopia was present in $64 \%$. In $18 \%$, no ocular weakness was manifest. The most frequent pattern of ocular weakness was isolated diplopia $(42 \%)$ or ptosis with diplopia (22\%). Ptosis was asymmetric in $92 \%$ and $\mathrm{ECW}$ in $9 \%$. A combination of horizontal and vertical diplopia was observed in $95 \%$ of patients with diplopia, purely horizontal diplopia was present in 5\% and purely vertical diplopia did not occur. In $82 \%$ of patients, diplopia occurred in more than one gaze direction at the first visit. Ocular weakness patterns at the first visit are reported in more detail in
Table 1

Baseline characteristics of 306 patients with MG included in this study

\begin{tabular}{lcc}
\hline & $\begin{array}{c}\text { Prospective } \\
\text { cohort } \\
\mathrm{N}=144\end{array}$ & $\begin{array}{c}\text { Retrospective } \\
\text { cohort } \\
\mathrm{N}=162\end{array}$ \\
\hline Age, y & $58.0 \pm 16.7$ & $57.1 \pm 19.6$ \\
Age at onset, y & $45.5 \pm 19.6$ & $43.6 \pm 20.5$ \\
Gender & $58(40)$ & $51(32)$ \\
$\quad$ Male & $86(60)$ & $111(68)$ \\
Female & & \\
Antibodies & $120(83)$ & $104(64)$ \\
AChR+ & $8(6)$ & $14(9)$ \\
MuSK+ & $16(11)$ & $44(27)$ \\
Seronegative & $84(58)$ & $54(33)$ \\
Immunotherapy & & \\
Thymectomy & $11(8)$ & $17(11)$ \\
$\quad$ Yes, with thymoma & $26(18)$ & $24(15)$ \\
Yes, without thymoma & $107(74)$ & $121(75)$ \\
$\quad$ No & $4.1 \pm 3.5$ & $3.3 \pm 1.7$ \\
MG-ADL & $6.6 \pm 4.7$ & $9.7 \pm 5.5$ \\
QMG &
\end{tabular}

Baseline characteristics, including MG-ADL and QMG scores at the first visit of 306 patients with MG included in this study are shown. Data are presented as number of patients (\%) for categorical variables and as mean \pm SD for continuous variables.

Table 2. None of the 20 healthy controls manifested diplopia during EOM fatigability testing.

\section{Changes in ocular weakness patterns over time}

146 patients had multiple visits during which ptosis and ECW were investigated. Forty percent of patients with initial symmetric ptosis developed asymmetric ptosis at the second visit (Fig. 1). The change from symmetric ptosis to asymmetric ptosis occurred significantly more often than the other way around $(p=0.0002)$. Only $1 \%$ of patients with initial asymmetric ptosis developed symmetric ptosis at the second visit. In 10\% of MG patients, the side of ptosis changed at the second visit. Changes in form of ptosis occurred less often in SNMG patients $(50 \%)$ than in $\operatorname{AChR}(70 \%)$ or MuSK patients $(69 \%)(p=0.038)$.

Eighty-three percent of patients with diplopia at the first visit manifested diplopia in other gaze directions at the next visit. Figure 2 provides an overview of the gaze directions in which diplopia was observed on the first and second visit in 3 MG patients that showed substantial changes in diplopia pattern. The time between visits was 4 weeks in these patients. Five percent of patients with initial symmetric ECW developed (temporary) asymmetric ECW at some point within their whole disease course. 
Table 2

Baseline ocular weakness patterns

\begin{tabular}{lc}
\hline & MG patients \\
& $\mathrm{N}=306$ \\
\hline Ptosis (P) & \\
Absent & $104(34)$ \\
Right eye & $102(33)$ \\
Left eye & $84(28)$ \\
Symmetric & $16(5)$ \\
ECW & $221(72)$ \\
Absent & $2(1)$ \\
Right eye & $6(2)$ \\
Left eye & $77(25)$ \\
Symmetric & \\
\hline & MG patients \\
& $\mathrm{N}=144^{\mathrm{a}}$ \\
\hline Diplopia (D) & $52(36)$ \\
Absent & $0(0)$ \\
Vertical & $5(3)$ \\
Horizontal & $87(61)$ \\
Both & $26(18)$ \\
No ocular weakness & \\
P only & $10(7)$ \\
D only & $50(35)$ \\
ECW only & $3(2)$ \\
P + D & $26(18)$ \\
P + ECW & $3(2)$ \\
D + ECW & $12(8)$ \\
P + D + ECW & $14(10)$ \\
\hline
\end{tabular}

The frequency of the occurrence of ptosis $(\mathrm{P})$, eye closure weakness (ECW) and diplopia (D) at the first visit are shown. In addition, the frequency of simultaneously present ocular symptoms is reported. Data are presented as number of patients (\%). ${ }^{\text {a }}$ Diplopia and combinations of ocular symptoms is shown of the prospective cohort of 144 MG patients.

\section{Provocation time required to develop ptosis or diplopia}

The mean time (in seconds) to diplopia was $11.6 \pm 14.0$ (Fig. 3) and the mean time to ptosis was $27.6 \pm 19.8$ (Fig. 4). Diplopia or ptosis manifested within 30 seconds in $87 \%$ and $58 \%$, respectively. The correlation between the lowest individual times to diplopia or ptosis and their respective MG-ADL items were $r=-0.340 ; p=0.001$ and $r=0.0407 ; p=0.132$, respectively. In other words, diplopia usually manifested within 30 seconds and a longer time to onset during examination correlated significantly with a lower patient-reported disease burden. In contrast, ptosis occurred almost as frequently within $0-30$ seconds as within 30-60 seconds and the time to ptosis during examination did not show a correlation to patient-reported limitations. Figure 5 shows that diplopia that manifested after 30 seconds was not associated with limitations in MG patients. The MG-

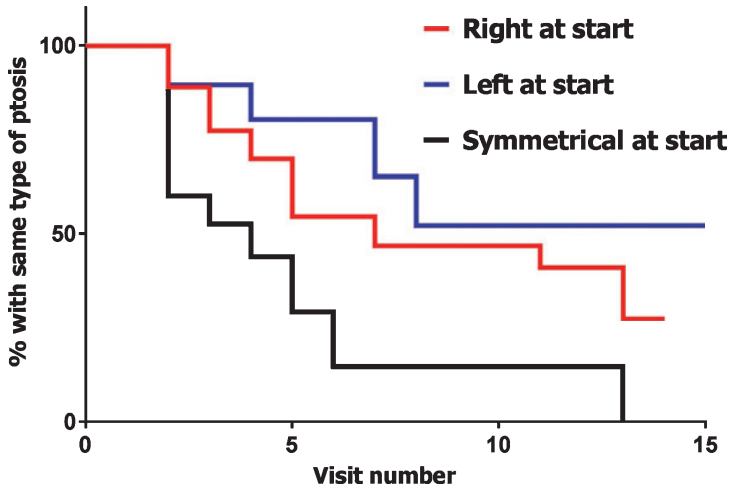

Fig. 1. Changes in type of ptosis. Kaplan-Meier survival curves indicate the change of the type of ptosis within 146 MG patients, subdivided by initial type of ptosis. Only changes from one side to another or from symmetric to asymmetric or vice versa were considered changes, the disappearance of ptosis was not considered as a change of type of ptosis. Both retro- and prospective data was used for these curves.

ADLdip was significantly correlated to the presence of diplopia $(p<0.001)$. However, 50 patients with diplopia during examination $(56 \%)$ did not report limitations due to diplopia.

\section{Clinical diplopia and the diplopia item of the $Q M G$}

Of the 92 patients with diplopia, 29 (32\%) did not manifest diplopia in the horizontal right gaze direction. Thus, the current diplopia item of the QMG would be considered normal in $32 \%$ of patients with diplopia. As we previously found that diplopia occurring after 30 seconds was both rare and of no major burden for the patient, we investigated which additional gaze directions gave the highest yield within 30 seconds of testing. By additionally testing the top left gaze direction for 30 seconds, 14 additional patients manifested diplopia (15\%). By also testing the bottom right gaze direction for 30 seconds, another 8 patients (9\%) manifested diplopia, thereby missing only 7 patients with overall diplopia (8\%), of which only 3 with relevant diplopia (manifesting within 30 seconds).

\section{DISCUSSION}

This is the first detailed study of fluctuations in ocular weakness in a large group of $306 \mathrm{MG}$ patients. Both EOM weakness and the side of ptosis frequently change over the course of the disease. In addition, diplopia mostly has both a horizontal and vertical 


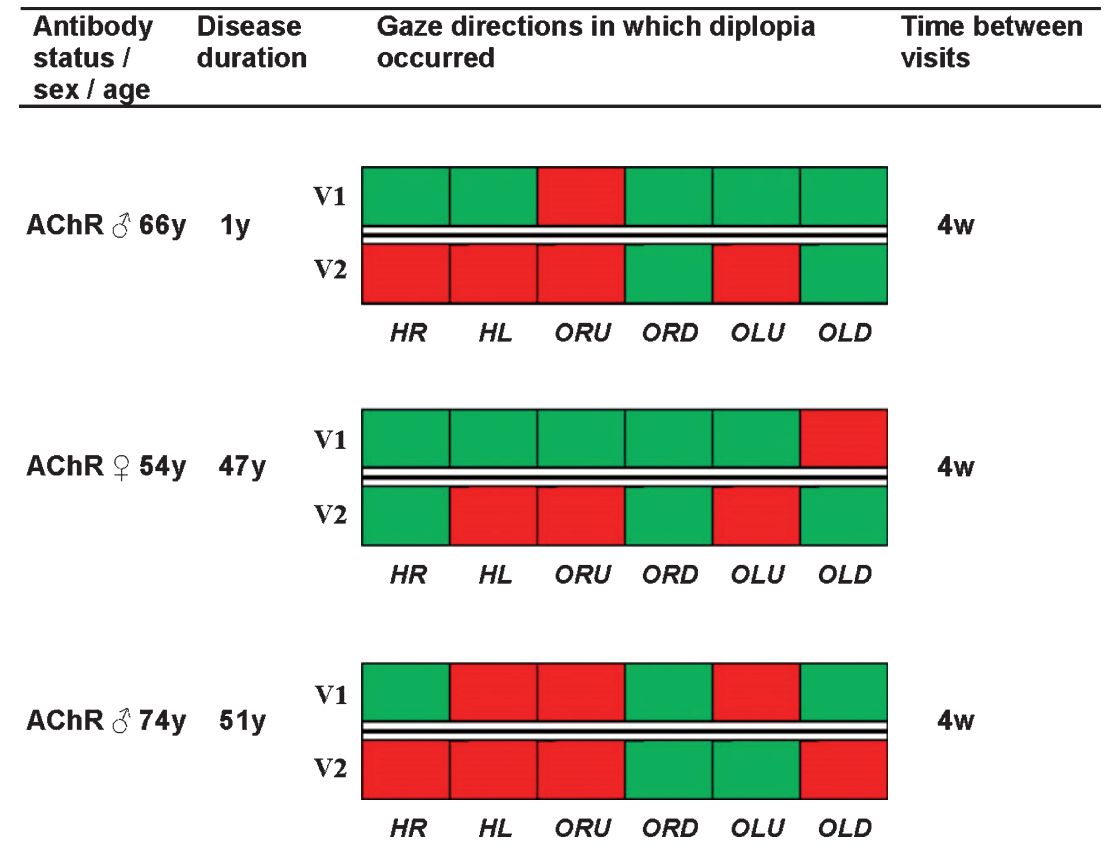

Fig. 2. Changes of diplopia patterns in individual MG patients. Baseline characteristics and an overview of gaze directions in which diplopia occurred of three MG patients that had a substantial change of diplopia pattern at the second visit. Red indicates that diplopia occurred in that gaze direction and green indicates that no diplopia occurred in that gaze direction. Abbreviations: $\mathrm{HR}=$ horizontal right, $\mathrm{HL}=$ horizontal left, $\mathrm{ORU}=$ oblique right upward, $\mathrm{ORD}=$ oblique right downward, $\mathrm{OLU}=$ oblique left upward, $\mathrm{OLD}=$ oblique left downward.

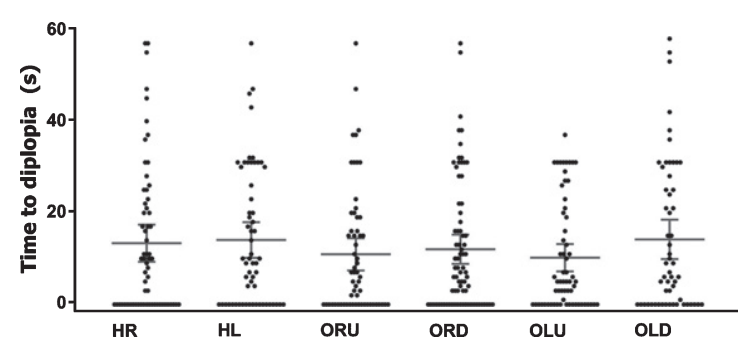

Fig. 3. Time to diplopia for each gaze direction. Scatter dot plots showing the time within which diplopia manifested after provocation for each gaze direction. Mean and 95\% CI are shown by lines and error bars. Dots at the bottom (0), indicate spontaneous diplopia. Data of patients without diplopia have been omitted. Abbreviations: $\mathrm{HR}=$ horizontal right, $\mathrm{HL}=$ horizontal left, $\mathrm{ORU}=$ oblique right upward, $\mathrm{ORD}=$ oblique right downward, $\mathrm{OLU}=$ oblique left upward, $\mathrm{OLD}=$ oblique left downward.

component and ptosis is most commonly asymmetrical or becomes so over time. Diplopia usually occurs within 30 seconds and time to diplopia is strongly correlated with MG-ADLdip. Ptosis takes longer to be provoked and is not correlated with patient-reported measures (MG-ADLpto).

Eighty-two percent of the MG patients in our prospective cohort had ocular symptoms at the first visit. This is in line with previous studies reporting that approximately $85 \%$ of MG patients had ocular

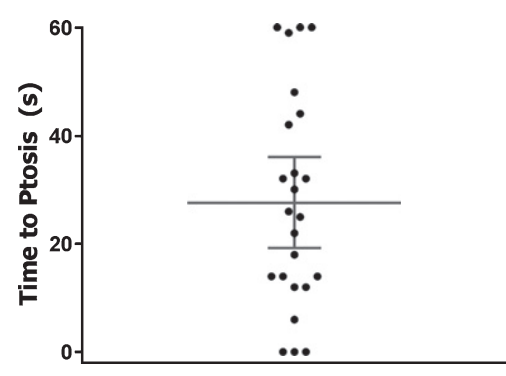

Fig. 4. Time to ptosis. Scatter dot plot showing the time within which ptosis manifested after provocation. The eye with the shortest time to diplopia is plotted here. Mean and $95 \% \mathrm{CI}$ are shown by lines and error bars. Dots at the bottom (0), indicate spontaneous ptosis. Data of patients without ptosis have been omitted.

symptoms at disease onset, while only $10-20 \%$ of patients had weakness of arms or legs initially [2, 11]. The fact that ocular muscles are predominantly affected clinically in MG may be caused by differences in neuromuscular junction (NMJ) morphology and physiology. Several features of the NMJs of EOMs may cause their increased susceptibility: less prominent synaptic folds, fewer postsynaptic AChRs, smaller motor units (around 4-12 muscle fibers compared to 400-600 in limb muscles), the 6-8 times higher innervation rate and the low expression of complement regulators [3, 12-17]. In addition to dif- 


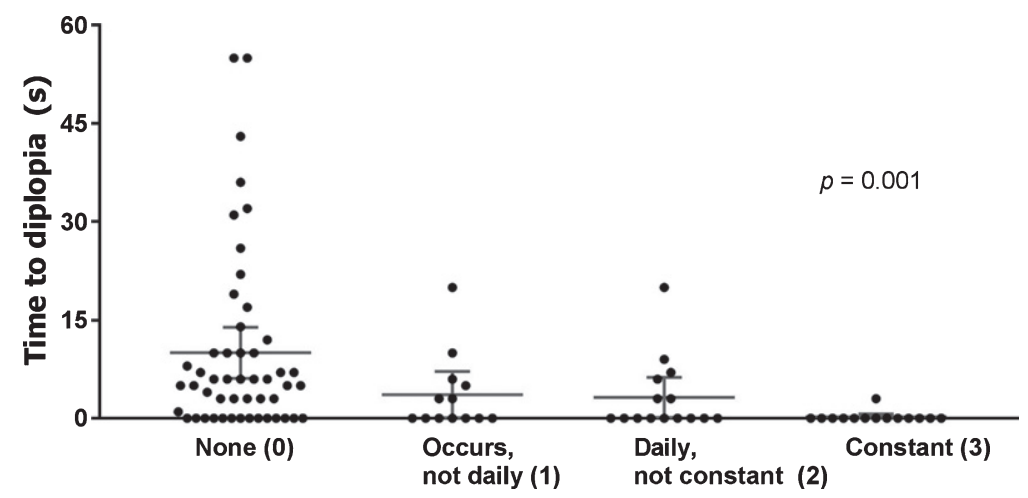

Fig. 5. Correlation time to diplopia and MG-ADL diplopia item. Scatter dot plots showing the time within which diplopia manifested after provocation, grouped according to the MG-ADL diplopia score of each MG patient (x-axis). Eight gaze directions were tested per patient, the gaze direction with the shortest time to diplopia is plotted here. Mean and 95\% CI are shown by lines and error bars. Dots at the bottom (0), indicate spontaneous diplopia. Data of patients without diplopia have been omitted.

ferences at the level of the NMJ, EOMs have distinct functional properties that may also play a role in the increased vulnerability of these muscles. Compared to limb muscles, EOMs are continuously active, have limited peak activity and due to the equal innervation of EOMs, a minor deficit in innervation can lead to diplopia as no compensation can occur [3]. However, an explanation for the frequent occurrence of changes in involved EOMs is currently lacking. In contrast to EOMs (or LPS), fluctuations in generalized weakness often affect muscles on both sides in a similar degree [1]. Future research investigating the differences between muscles should also focus on clarifying why changes in affected EOMs occur more frequently than changes in side of affected limbs.

In our prospective cohort of MG patients, $64 \%$ manifested diplopia during EOM fatigability testing. None of the 20 healthy controls manifested diplopia during this test. This was in line with earlier studies showing that EOMs are relatively fatigue resistant in healthy subjects $[18,19]$. Furthermore, our findings show that the gaze directions in which diplopia occurs frequently change between visits. In $83 \%$ of MG patients diplopia in other gaze directions was found at the second visit. In patients who developed diplopia, it usually manifested within 30 seconds. When diplopia was present, double vision contained both a vertical and horizontal component in most cases (95\%).

Diplopia manifested within 30 seconds in $87 \%$ and a strong correlation was found between time to diplopia and MG-ADLdip, suggesting that time to diplopia is a good marker for clinical severity. However, 54\% of patients with observed diplopia (in $48 \%$ occurring within 30 seconds) did not report limitations due to diplopia (MG-ADLdip $=0$ ). We hypothesize that the discrepancy between physicianreported diplopia by testing EOM fatigability and the patient-reported burden due to diplopia is caused by adaptive mechanisms such as turning the head into the desired direction straight away without (first) moving the eyes in that direction.

When ptosis is present, it is usually asymmetric. In those patients who present with symmetric ptosis, it usually becomes asymmetric within a few visits. In patients with asymmetric ptosis, the worst affected side frequently changes. These features should be helpful in the distinction from other causes of ptosis, such as cranial nerve palsy or chronic progressive external ophtalmoplegia, which are not expected to show similar fluctuations. Interestingly, changes in form of ptosis occurred less often in SNMG patients than in AChR or MuSK MG patients. To our knowledge, it has not earlier been described that SNMG patients show less disease fluctuations. In contrast to diplopia, ptosis was provoked within 30 seconds in only a minority of patients (42\%), suggesting that longer testing is needed for an optimal evaluation of ptosis. Our findings differ from that of a study by Burns et al. on the Myasthenia Gravis Composite (MGC) scale, who reported that $<1 \%$ of ocular score times were between 46 and 60 seconds compared to $21 \%$ in our cohort [20].

In contrast to diplopia, no significant correlation was found between time to ptosis and the clinical burden as quantified in the MG-ADLpto. We hypothesize that the reason for this lack of correlation is that in daily life, the provocation of ptosis (by looking upward for a prolonged time) occurs less frequently and could be diminished by adaptive mechanisms, such as watching television with 
the eyes in a slightly depressed position. In addition, subtle forms of ptosis are less likely to affect quality of life as long as the upper eyelid does not impair vision.

The strength of this study is the systematic evaluation of ocular weakness patterns in a large cohort of clinically well-defined MG patients. However, the study has a number of limitations. Patients were included from a single center and as this is a tertiary referral center, our study may not fully reflect the total MG population due to referral bias. Moreover, our testing method for EOM weakness was not reliable enough to identify the specific EOMs involved and did not provide quantitative data on the deviation of the eye axes (i.e. angle between the axes), as can be measured by ophthalmologic tools such as the Hess chart. However, the Hess chart does not provide data on the time required to develop diplopia. As muscle fatigability rather than weakness is the hallmark of MG, we focused on 'provocation time' instead of the quantitative deviation of the eye axes at a single time point. Indeed, our data show that time to diplopia is strongly correlated with clinical, patient-reported severity of diplopia. In addition, the testing method described here is applicable by neurologists that treat MG patients without the use of advanced equipment. Finally, the retrospective analysis of the occurrence and symmetry of ptosis and ECW over time is a limitation of this study. However, retrospective analyses allowed us to study a far larger number of patients and patient visits and provided us with a longer time period over which changes in ptosis and ECW could be investigated.

In conclusion, ocular weakness patterns frequently change during the disease course, even within short intervals of 4 weeks. In $10-17 \%$ of patients, muscle weakness remains clinically localized to the ocular muscles during the entire disease course (ocular MG; OMG) [2, 3]. As both laboratory and neurophysiological tests have a lower sensitivity and specificity in OMG, the diagnosis is more difficult to make in this group of patients and often relies solely on clinical grounds [21]. Here, we described typical ocular weakness patterns to aid clinical recognition of MG: changes in the gaze directions in which diplopia occurs or in ptosis side and the presence of both horizontal and vertical diplopia or asymmetrical ptosis. In diagnostically challenging cases, we recommend testing ptosis and diplopia in multiple gaze directions for 30-60 seconds during at least two follow-up visits to maximize the chance of observing changes in ocular weakness patterns.

\section{STUDY FUNDING}

This study was supported by funding of the Prinses Beatrix Spierfonds, and the MD/PhD-program for Medicine students of the Leiden University Medical Center.

\section{ETHICAL PUBLICATION STATEMENT}

We confirm that we have read the Journal's position on issues involved in ethical publication and affirm that this report is consistent with those guidelines.

\section{DISCLOSURES}

R.H.P. de Meel reports no disclosures.

W.F. Raadsheer reports no disclosures.

E.W. van Zwet reports no disclosures.

M.R. Tannemaat reports no disclosures.

J.J.G.M. Verschuuren has been involved with MG research sponsored by the Prinses Beatrix Fonds, NIH, FP7 European grant (\#602420), consultancies for Argen-X, Alexion and Rapharma. The LUMC received royalties from IBL for antibody tests. All reimbursements were received by the LUMC.

\section{REFERENCES}

[1] Meriggioli MN, Sanders DB. Autoimmune myasthenia gravis: Emerging clinical and biological heterogeneity. The Lancet Neurology. 2009;8:475-90.

[2] Grob D, Brunner N, Namba T, Pagala M. Lifetime course of myasthenia gravis. Muscle \& Nerve. 2008;37:141-9.

[3] Oosterhuis HJ. The ocular signs and symptoms of myasthenia gravis. Documenta Ophthalmologica Advances in Ophthalmology. 1982;52:363-78.

[4] Cleary M, Williams GJ, Metcalfe RA. The pattern of extra-ocular muscle involvement in ocular myasthenia. Strabismus. 2008;16:11-8.

[5] Almog Y, Ben-David M, Nemet AY. Inferior oblique muscle paresis as a sign of myasthenia gravis. Journal of Clinical Neuroscience: Official Journal of the Neurosurgical Society of Australasia. 2016;25:50-3.

[6] de Meel RHP, Verschuuren J, Tannemaat MR. Distinct representation of muscle weakness in QMG and MG-ADL. The Lancet Neurology. 2018;17:204-5.

[7] Wong SH. Rating scale for ocular myasthenia gravis: A call to action! Journal of Neuro-Ophthalmology: The Official Journal of the North American Neuro-Ophthalmology Society. 2018;38:138-9.

[8] Y. S, H.W. L, M.H. K, M. S, H. C and J.H. K, Normal range of eye movement and its relationship to age. Acta Ophthalmologica. 2016;94.

[9] Urist MJ. A lateral version light-reflex test. American Journal of Ophthalmology. 1967;63:808-15.

[10] Clark RA, Isenberg SJ. The range of ocular movements decreases with aging. Journal of AAPOS: The Official 
Publication of the American Association for Pediatric Ophthalmology and Strabismus. 2001;5:26-30.

[11] Beekman R, Kuks JB, Oosterhuis HJ. Myasthenia gravis: Diagnosis and follow-up of 100 consecutive patients. Journal of Neurology. 1997;244:112-8.

[12] Soltys J, Gong B, Kaminski HJ, Zhou Y, Kusner LL. Extraocular muscle susceptibility to myasthenia gravis: Unique immunological environment? Annals of the New York Academy of Sciences. 2008;1132:220-4.

[13] Luchanok U, Kaminski HJ. Ocular myasthenia: Diagnostic and treatment recommendations and the evidence base. Current Opinion in Neurology. 2008;21:8-15.

[14] Kaminski HJ, Li Z, Richmonds C, Lin F, Medof ME. Complement regulators in extraocular muscle and experimental autoimmune myasthenia gravis. Experimental Neurology. 2004;189:333-42.

[15] Liu R, Xu H, Wang G, et al. Extraocular muscle characteristics related to myasthenia gravis susceptibility. PloS One. 2013;8:e55611.

[16] Horton RM, Manfredi AA, Conti-Tronconi BM. The 'embryonic' gamma subunit of the nicotinic acetylcholine receptor is expressed in adult extraocular muscle. Neurology. 1993;43:983-6.
[17] Gunji K, Skolnick C, Bednarczuk T, et al. Eye muscle antibodies in patients with ocular myasthenia gravis: Possible mechanism for eye muscle inflammation in acetylcholinereceptor antibody-negative patients. Clinical Immunology and Immunopathology. 1998;87:276-81.

[18] Kaminski HJ, Richmonds CR. Extraocular muscle fatigue. Annals of the New York Academy of Sciences. 2002;956:397-8.

[19] Fuchs AF, Binder MD. Fatigue resistance of human extraocular muscles. Journal of Neurophysiology. 1983;49:28-34.

[20] Burns TM, Conaway M, Sanders DB. The MG Composite: A valid and reliable outcome measure for myasthenia gravis. Neurology. 2010;74:1434-40.

[21] Benatar M. A systematic review of diagnostic studies in myasthenia gravis. Neuromuscular Disorders: NMD. 2006;16:459-67. 